\section{Vascular endothelial growth factor profile and Vitamin D level in Systemic Sclerosis Egyptian patients}

\author{
Nermin H. El Gharbawy ${ }^{1}$, Dina S. Sheha ${ }^{2}$, Somia A. \\ Bawady ${ }^{3}$, Sally A. EL Leithy ${ }^{1}$
}

${ }^{1}$ Department of Physical Medicine, Rheumatology \& Rehabilitation, Faculty of Medicine, AinShams University, Cairo, Egypt.

${ }^{2}$ Department of Internal Medicine, Allergy \& Clinical Immunology, Faculty of Medicine, AinShams University, Cairo, Egypt.

${ }^{3}$ Department of Clinical Pathology, Faculty of Medicine, Ain Shams University, Cairo, Egypt.
The Egyptian Journal of Immunology Volume 28 (3), 2021: 168-175. www.Ejimmunology.org
Corresponding author: Dina S. Sheha, Department of Internal Medicine, Faculty of Medicine, AinShams University, Abbassia, Cairo, Egypt. Postal code: 11566.

Email: DinaSheha@med.asu.edu.eg.

\begin{abstract}
Vascular endothelial growth factor (VEGF) was described as a potentially important driver of systemic sclerosis (SSc) pathogenesis. Additionally, recent literature elucidated that vitamin D serum level was found to be significantly lower in SSc patients in comparison to healthy individuals. The aim of the current study was to evaluate serum level of VEGF and its correlation with clinical features and vitamin D level in systemic sclerosis (SSc) Egyptian patients. This current case control study included 30 female SSc patients and 20 healthy controls. VEGF level was measured by ELISA. Serum level of $25-\mathrm{OH}$ vitamin D was measured by electrochemiluminescence. Nailfold video capillaroscopy and modified Rodnan skin score (mRSS) were assessed. Thirty SSc female patients were included in the study, 13 patients had diffuse cutaneous SSc. The mean age of the patients' group was $49.3 \pm 4.3$ years, and the mean serum VEGF level was $3445.9 \pm 1183 \mathrm{ng} / \mathrm{dl}$. The mean serum level of vitamin D was $15.57 \pm 9.9 \mathrm{ng} / \mathrm{ml}$ in SSc patients and $30.6 \pm 2.26$ in the controls. There was a significant association between high level of VEGF and hypovitaminosis D. Serum level of VEGF positively correlated with nailfold capillaroscopy changes and mRSS. In conclusion, high level of VEGF is associated with hypovitaminosis D, suggesting a role of vitamin D in SSc pathogenesis. VEGF levels correlate positively with nailfold capillaroscopy changes and extent of skin involvement.
\end{abstract}

Keywords: SSc (systemic sclerosis), VEGF (vascular endothelial growth factor, vasculopathy, cutaneous fibrosis, Vitamin D, nailfold capillaroscopy

Date received: 11 April 2021; accepted: 22 July 2021

\section{Introduction}

Systemic sclerosis (SSc) is a rare multisystem autoinmune disease of unknown etiology and complex pathophysiology ${ }^{1} \quad$ Multiple environmental and genetic factors seem to interact in triggering SSc and its complications. ${ }^{2}$ The pathological hallmarks of systemic sclerosis constitute of vasculopathy, autoimmunity and tissue remodeling manifestations such as circulating antibodies, Raynaud's disease and 
cutaneous fibrosis. ${ }^{3}$ Campbell and Le Roy were the first to suggest that vasculopathy is an important driver of autoimmunity and tissue remodeling. ${ }^{4}$ Treatment is focused on the control of symptoms and specific organ involvement considering the balance between the benefit and toxicity of each treatment. ${ }^{5}$

Endothelial injury is an important initiating event in vasculopathy in SSc. Increased vascular permeability, endothelial dysfunction and perivasular inflammatory cell infiltrate predates the development of tissue fibrosis. ${ }^{6}$ Vascular endothelial growth factor (VEGF) was described as a potentially important driver of SSC pathogenesis. ${ }^{7}$ It is considered as a vascular permeability factor which has both mitiogenic and angiogenic properties. ${ }^{8}$

Authors identified high levels in both early and established SSC as it has role in vasculopathy and fibrosis. ${ }^{9}$ Hypoxia is a major driver for expression of VEGF; while other cytokines and growth factors can enhance VEGF signaling. VEGF expression may enhance cytokines and growth factor signaling which could have effective implications on SSC pathogenesis. ${ }^{10}$ Although data denotes a possible role of chemokines such as soluble fractalkine $\mathrm{CX} 3 \mathrm{CL} 1$ in the pathogenesis of $\mathrm{SSc}^{11}$ VEGF remains the most potent angiogenic molecule involved in vasculopathy in those patients. ${ }^{12}$

Vitamin $D$ is a steroid hormone responsible for regulation of bone metabolism and calcium homeostasis. ${ }^{13}$ Vitamin $D$ has predominant immunomodulatory effects as it inhibits proinflammmatory cytokines and stimulates anti- inflammatory cytokines through Vitamin D receptors present on antigen presenting cells, natural killer (NK) cells, activated $T$ and $B$ lymphocytes. Also Vitamin $D$ stimulates the action of regulatory $T$ cells and inhibits -helper 17 cells. $^{14}$ Therefore Vitamin D seems to be protective against the development of autoimmunity enhancing the evidence of its immunomodulatory effect in the adaptive immune response. ${ }^{15}$ Vitamin D serum level was found to be significantly lower in SSc patients in comparison to healthy individuals. ${ }^{16}$ However, hypovitaminosis D in SSc pathophsiology is not yet well established in Egyptian patients. ${ }^{17}$ In the current study, we aimed to evaluate the VEGF profile and serum level of Vitamin D in SSC patients and its correlation with the clinical features and serum level of Vitamin D in SSC Egyptian patients.

\section{Patients and Methods}

\section{Patients, Study Design}

The current study is a case control study that recruited $30 \mathrm{SSc}$ patients from the Rheumatology and rehabilitation outpatient clinic of Ain Shams University Hospitals. By using PASS 11 program for sample size calculation, sample size of 30 patients and 20 controls can detect the difference in vitamin $D$ levels between the two groups with power $80 \%$ setting alpha error at 0.05 . The patients fulfilled the 2013 American college of Rheumatology/European league against Rheumatism for SSC ${ }^{18}$. Patients with overlapping syndromes, cancer and active infections were excluded. Informed written consent was obtained from all individuals participating in the study. The study was approved by the Research ethics committee of AinShams University and it was carried out in accordance with The Code of Ethics of the World Medical Association (Declaration of Helsinki).

\section{Clinical Assessment}

Patients were examined to assess the lesions in target organs in SSc. The regular use of sunblock was also assessed. Disease duration was defined as the time interval since the onset of Raynaud's phenomenon or skin symptoms. Disease Subtype was classified into limited cutaneous SSc and diffuse cutaneous SSc ${ }^{19}$. The severity of skin disease was evaluated using modified Rodnan skin score (mRSS). ${ }^{20}$

Blood samples were collected from all participants

A $5 \mathrm{ml}$ venous blood sample was withdrawn into a plain vacutainer. After clotting, samples were centrifuged and separated serum was aliquoted and stored at $-20^{\circ} \mathrm{C}$ to be used for measurement of VEGF, and $25(\mathrm{OH})$ vitamin D. 
Hemolysed samples were discarded. Repeated freezing and thawing was avoided.

\section{Vascular endothelial Growth Factor Measurement}

VEGF concentration was measured using a commercially available enzyme-linked immunosorbent assay (ELISA) kit supplied by (Bioassay Technology Laboratory, Shanghai, China) using sandwich ELISA technique.

\section{Vitamin D measurement}

Vitamine $D$ levels were measured during spring and summer, 2019, so as to limit the effect of seasonal variations in photosynthesis of Vitamin D. $25(\mathrm{OH})$ vitamin $D$ was measured by electochemiluminescence immunoassay (ms_07464215190V2.0) on cobas e 411 (Roche Diagnostics $\mathrm{GmbH}$, SandhoferStrasse 116, D68305 Mannheim). Vitamin D deficiency was defined as serum level lower than $10 \mathrm{ng} / \mathrm{ml}$ and insufficiency as serum level between 10 and 30 $\mathrm{ng} / \mathrm{ml}$ in SSc patients. ${ }^{21}$

\section{Nailfold video capillaroscopy}

It was performed for all patients using (Optilia Digital Capillaroscopy System, Sweden). Patients were asked to avoid vasoactive drugs during at least five half lives for each medication, manicure, nail polish and cuticulate trauma for at least 3 weeks. Also, patients were asked not to take caffeinated drinks for at least 4 hours before NVC and to remain at least 15 minutes in temperature 22-23 degree. Nail fold of $2^{\text {nd }}, 3^{\text {rd }}$, $4^{\text {th }}$, and $5^{\text {th }}$ were examined using video capillaroscope equipped with $\times 200$ magnification contact lens. Four consecutive fields extending over $1 \mathrm{~mm}$ in the middle of nail fold were studied per finger ${ }^{22}$. The following definitions were used for the qualitative assessment of NVC patterns. The early pattern: the combination of few enlarged/giant capillaries, few capillary microhemorrhages, a relatively well preserved capillary distribution and no evident loss of capillaries. The active pattern frequent giant capillaries, frequent capillary microhemorrhages, moderate loss of capillaries, mild disorganization of the capillary architecture, and absent or mild ramified capillaries. The late pattern: irregular enlargement of capillaries, few or absent giant capillaries and microhemorrhages, severe loss of capillaries with large avascular areas, disorganization of the normal capillary array, and ramified/bushy capillaries ${ }^{23}$.

\section{Statistical analysis}

The collected data was revised, coded, tabulated and introduced to a PC using Statistical Package for Social Science version 20 (SPSS-V20) USA. Data was presented and suitable analysis was done according to type of data obtained for each parameter. Mean \pm SD and range for numerical data, frequency and percentage for non-numerical data. Student-T test was used to compare between two groups in quantitative data. Linear correlation coefficient was used to assess the strength of association between non-parametric variables in the same group. ANOVA test was used for comparison of different times in the same group in quantitative data. Results were considered significant at $P \leq 0.05$.

\section{Results}

Demographic and clinical data of SSc patients and controls is demonstrated in table 1 .

Nailfold capillaroscopy showed late changes in 14 patients (46.6\%), active changes in 11 patients (36.6\%) and early changes in 2 patients (6.67\%). The serum level of vitamin D in SSc patients ranged from 5.5 to $36 \mathrm{ng} / \mathrm{ml}$ with mean $15 \pm 9.9 \mathrm{ng} / \mathrm{ml}$ while in the control group ranged from 27.7 to $35 \mathrm{ng} / \mathrm{ml}$ with mean $30.6 \pm 2.26$ $\mathrm{ng} / \mathrm{ml}$. Vitamin D deficiency was observed in 16 patients and was insufficient in 4 patients. Healthy controls did not have vitamin D deficiency and 12 had vitamin D insufficiency. Sixteen patients had VEGF level above 3500 $\mathrm{ng} / \mathrm{dl}$ and 9 patients ranged from 3250-3400 $\mathrm{ng} / \mathrm{dl}$ (table 1). 
Table 1. Demographic and clinical data of systemic sclerosis patients and controls

\begin{tabular}{|c|c|c|c|c|}
\hline & \multicolumn{2}{|c|}{$\begin{array}{c}\text { Patient } \\
\text { Mean士 (SD) }\end{array}$} & \multicolumn{2}{|c|}{$\begin{array}{c}\text { Control } \\
\text { Mean } \pm(S D)\end{array}$} \\
\hline Age (years) & \multicolumn{2}{|c|}{$49.3 \pm 4.3$} & \multicolumn{2}{|c|}{$48.9 \pm 3.44$} \\
\hline Duration of disease (years) & \multicolumn{2}{|c|}{$9.9 \pm 3.1$} & \multicolumn{2}{|c|}{-} \\
\hline \multirow{3}{*}{ Skin Involvement } & & $\%$ & \multirow{3}{*}{\multicolumn{2}{|c|}{ - }} \\
\hline & limited & 56.6 & & \\
\hline & diffuse & 43.3 & & \\
\hline ESR level (mm/hr) & \multicolumn{2}{|c|}{$37 \pm 10.1$} & \multicolumn{2}{|c|}{$8 \pm 2$} \\
\hline \multirow{4}{*}{ Vit D level (ng/ml) } & \multicolumn{2}{|r|}{$\mathrm{N}$} & & $\mathrm{N}$ \\
\hline & \multicolumn{2}{|l|}{ Normal } & Normal & 8 \\
\hline & Insufficiency & 9 & Insufficiency & 12 \\
\hline & Deficiency & 16 & Deficiency & - \\
\hline VEGF level (ng/dl) & \multicolumn{2}{|c|}{$3445.9 \pm 1183.5$} & \multicolumn{2}{|c|}{$1530 \pm 437$} \\
\hline Modified Rodnan skin score & \multicolumn{2}{|c|}{$12.16 \pm 3.75$} & \multicolumn{2}{|c|}{-} \\
\hline \multirow{5}{*}{ Nail fold Capillaroscopy } & & $\%$ & & \\
\hline & Normal & 10 & & \\
\hline & Early & 6.67 & & \\
\hline & Active & 36.67 & \multirow{2}{*}{\multicolumn{2}{|c|}{-}} \\
\hline & Late & 46.67 & & \\
\hline
\end{tabular}

Vitamin D serum level was significantly lower in SSc patients in comparison with the control group $(P<0.001)$. Serum level of VEGF was significantly higher in SSc patients in comparison to controls $(P<0.001)$ (table 2$)$. There was a statistically highly significant relation between disease duration and each of nailfold capillaroscopy changes and mRSS. There was a significant inverse relationship between vitamin $D$ deficiency and disease duration and
ESR level. mRSS score was significantly higher in vitamin D deficient patients .

We found a significant relation between vitamin $D$ deficiency and disease duration $(P<$ 0.036). There was a highly significant relation between vitamin $D$ deficiency and each of mRSS $(P<0.001)$, ESR level $(P<0.001)$ and significant relation with nailfold capillaroscopy changes $(P<$ 0.001).

Table 2. Serum level of VEGF and Vitamin D in systemic sclerosis patients and controls.

\begin{tabular}{lccccc} 
& \multicolumn{2}{c}{ Patients } & \multicolumn{2}{c}{ Control } & \multirow{2}{*}{$P$ value } \\
\cline { 2 - 5 } & Mean & SD & Mean & SD & $<0.001$ \\
\hline Vit D level $(\mathrm{ng} / \mathrm{ml})$ & 15.57 & 9.9 & 30.6 & 2.26 & $<0.001$
\end{tabular}

Vit $\mathrm{D}$ : vitamin $\mathrm{D}$, ng: nanogram, VEGF: vascular endothelial growth factor, $P \leq 0.05$ is significant

There was a highly significant correlation between VEGF level and disease duration ( $r=$ $0.678, P<0.01)$, ESR level $(r=0.842, P<0.01)$ and mRSS ( $r=0.743, P<0.001)$. There was a significant relation between VEGF levels and nailfold capillaroscopy changes $(P<0.001)$. There was a highly significant correlation between high VEGF with low vitamin D level in SSC patients $(r=-0.09, P<0.001)$ (table 3$)$, (Figure 1$)$. 
Table 3. Correlation between VEGF level and disease duration, ESR level, mRSS, vitamin D level and nail capillaroscopy changes.

\begin{tabular}{lcc}
\hline & \multicolumn{2}{c}{ VEGF } \\
\cline { 2 - 3 } & $\mathrm{R}$ & $P$ value \\
\hline Disease Duration (years) & 0.678 & 0.01 \\
\hline ESR level (mm/hr) & 0.842 & 0.01 \\
\hline Modified Rodnan skin score & 0.743 & $<0.001$ \\
\hline Vit D Deficiency (ng/ml) & -0.09 & $<0.001$ \\
\hline Nail fold capillaroscopy & & $<0.001$
\end{tabular}

Spearman's Rho correlation test, $P<0.05$ is significant.

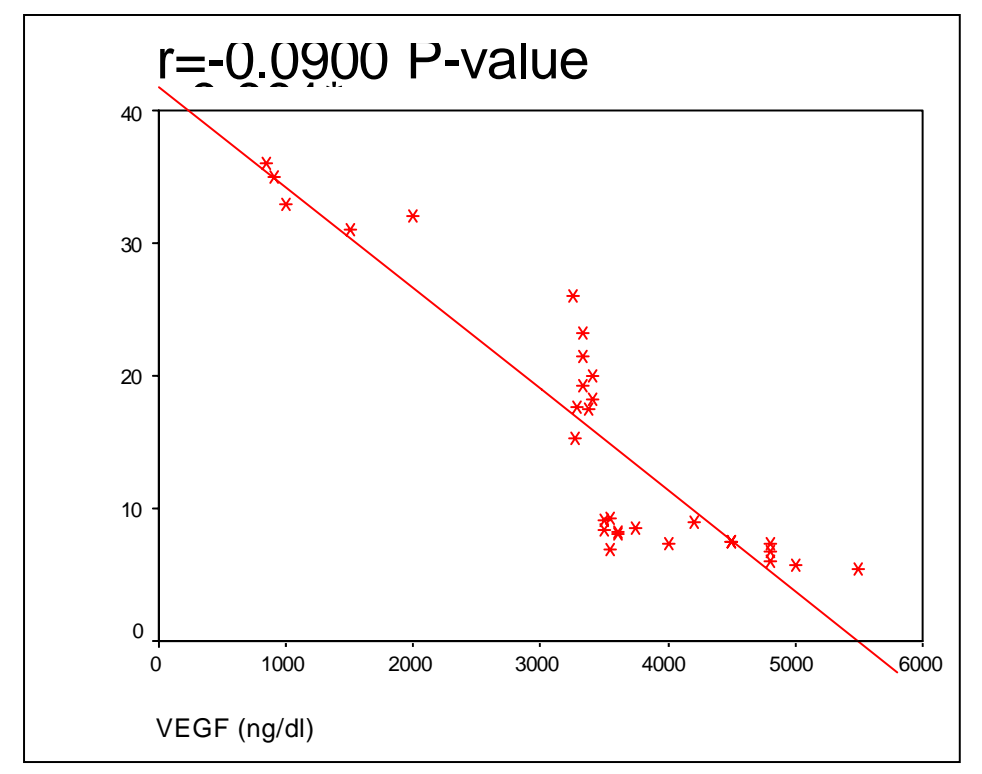

Figure 1. Correlation between VEGF profile and Vitamin D level in Systemic sclerosis patients. Normal range of vit $D$ : serum level more than $30 \mathrm{ng} / \mathrm{ml}$, vitamin $D$ deficiency: serum level lower than $10 \mathrm{ng} / \mathrm{ml}$ and vit $D$ insufficiency: serum level between 10 and $30 \mathrm{ng} / \mathrm{ml}$.

\section{Discussion}

The pathological hallmarks of SSc constitute an inter-related triad of autoimmunity, vasculopathy and tissue remodeling. Many signaling mediators have been implicated in SSC pathology. $^{24}$

VEGF plays an important role in SSC pathogenesis. Previous studies could not elucidate if low levels of vitamin D is a cause, potentiating factor or a consequence of SSc. ${ }^{25}$ The current study evaluated the serum level of
VEGF and its correlation with serum level of vitamin D and clinical features in SSc patients.

There was no statistical difference between patients and control groups as regards age and sex, which is in agreement with Hax et $a l .{ }^{16}$. We found low level of vitamin D in SSc patients compared to healthy controls, which is in agreement with published literature. ${ }^{17}$ Additionally, a study published in 2009 including 90 SSc patients from Northern France and 66 from Southern Italy showed that vitamin $D$ deficiency rates were very high ${ }^{26}$. The current 
study demonstrated high level of VEGF in SSC patients and normal level in the control group which is in accordance with another study which identified high levels of circulating VEGF in both early and established SSc patients ${ }^{27}$. We found a significant relation between disease duration and mRSS which may be due to the presence of high level of VEGF while other studies suggested skin improvement over time, which could be related to decline in serum levels of T helper 2 cytokines. ${ }^{28}$ Also, there was a statistically significant relation between disease duration and nailfold capillaroscopy changes which was demonstrated in a 20 years prospective study that noted transition from giant capillaries to capillary loss in nailfold capillaroscopy. ${ }^{29}$ The principle pathological cause is tissue hypoxia which is further exacerbated by oxygen demands of inflammatory milieu and impaired oxygen diffusion. ${ }^{30}$ A meta-analysis study demonstrated no correlation between vitamin $D$ level and mRSS, which could be explained by the immunomodulatory effect of vitamin $D$ which causes inhibition of Th1 cytokines, reduction of proinflammatory and increase of antiinflammatory cytokines postulating an important factor for profibrotic effect. ${ }^{14}$ We reported a significant correlation between low vitamin $D$ levels and mRSS which was demonstrated by authors. ${ }^{14}$ and was explained by the absence of immunomodulatory effect of vitamin $D$. There was a positive relation between VEGF and nailfold capillaroscopy changes which was in accordance with published literature that explained that VEGF upregulation does not sufficiently stimulate vasculogenesis. ${ }^{22}$ Prolonged overexpression of VEGF may have deleterious effects on the vascular network implicating vessel morophology. ${ }^{22}$ There was a highly significant correlation between VEGF and mRSS, which agrees with a study by Maurer et al., where serum levels of pan VEGF and skin score were supported by demonstration of increased collagen induction in SSc dermal fibroblasts in response to VEGF. ${ }^{31}$ Additionally, another study found pan VEGF is over expressed in non lesional skin predating the onset of fibrosis implicating VEGF is an early signaling protein in fibrosis ${ }^{10}$. There was a statistical correlation between hypovitaminosis $D$ levels and high VEGF in the present study, and a recent study correlated vitamin D level and cytokines profile ${ }^{16}$, concluding that normal level of vitamin D caused inhibition of Th1 cells, reduction of proinflammatory and increase in antiinflammatory cytokines levels but the study concluded that there is no association between cytokine profile and vitamin D level. It was supposed that the lack of difference in cytokine profile may be due to heterogenecity within SSC population, influence of long disease duration, systemic treatment and level of disease duration. ${ }^{32}$ One limitation of the current study is the small sample size and that recruited SSc patients were from a single-center. Additionally, the mean disease duration of participants was 10 years, thus the study involved mainly late patients with no active skin involvement but rather residual damage.

In conclusion, patients with SSc present with hypovitaminosis D, high levels of VEGF and nailfold changes. Hypovitaminosis $D$ and disease duration are important factors in the pathogenesis of SSc. High VEGF level correlates with hypovitaminosis $D$ which could be related to the absence of vitamin D immunomodulatory effect.

\section{Acknowledgments:}

We would like to thank all patients and controls who participated in the study

\section{Author Contributions}

N.G who was responsible for conception and design of the study, data acquisition, analysis and writing the original draft. D.S for data curation, formal analysis, manuscript editing, manuscript preparation and review corresponding. S.B was responsible for data acquisition, analysis, methodology and manuscript revision and S.L who performed design of the study, literature search, data analysis, manuscript preparation and writing the original draft.

\section{Declaration of Conflicting Interests}

The author(s) declared no potential conflicts of interest with respect to the research, authorship, and/or publication of this article. 


\section{Funding}

This research did not receive any specific grant from funding agencies in the public, commercial, or notfor-profit sectors.

\section{Ethical approval}

The study was approved by the Research ethics committee of AinShams University and it was carried out in accordance with The Code of Ethics of the World Medical Association (Declaration of Helsinki).

\section{Informed consent}

A signed consent form was obtained from each study participant.

\section{References}

1. Mo C, Zeng Z, Deng Q, et al. (2018). imbalance between $t$ helper 17 and regulatory $T$ cell subsets plays a significant role in the pathogenesis of systemic sclerosis. Biomed Pharmacother. 108:177-183.

2. Flower VA, Barratt SL, Ward S, et al. (2019). The Role of Vascular Endothelial Growth Factor in Systemic Sclerosis. Curr Rheumatol Rev. 15(2):99109.

3. Murdaca G, Contatore M, Gulli R, et al. (2016). Genetic factors and systemic sclerosis. Autoimmune Rev. 15:427-432.

4. Campbell PM, LeRoy EC (1975). Pathogenesis of systemic sclerosis: a vascular hypothesis. Seminars in arthritisand rheumatism. 4(4):35168.

5. Kowal-Bielecka O, Fransen J et al.(2017). Update of EULAR recommendations for treatment of systemic sclerosis. Ann Rheum Dis. 76:1327-1339.

6. Prescott RJ, Freemont AJ, Jones CJ, et al. (1992). Sequential dermal microvascular and perivascular changesin the development of scleroderma.The Journal of Pathology. 166(3):255-63.

7. Distler O, Del Rosso A, Giacomelli R et al (2002). Angiogenic and angiostatic factorsin systemic sclerosis: increased levels of vascular endothelial growth factor are a feature of the earliest disease stages and are associated with the absence of fingertip ulcers. Arthritis Research. 4(6):R11.

8. Leung DW, Cachianes G, Kuang WJ, et al. (1989). Vascular endothelial growth factor is a secreted angiogenic mitogen. Science. 246(4935):1306-9.

9. De Santis M, Ceribelli A, Cavacicchi F, et al (2016). Nailfold videocapillaroscopy and serum VEGF levels in scleroderma are associated with internal organ involvement. Auto-immunity highlights. $7(1): 5$.

10. Distler O, Distler JH, Scheid A et al (2004). Uncontrolled expression of vascular endothelial growth factor and its receptors leads to insufficient skin angiogenesis in patients with systemic sclerosis. Circulation Research. 95(1):109-16.

11. M. ElSergany, A. Shahba, M. Ghazy, M.M. et al. (2011). Increased expression of soluble fractalkine (CX3CL1) in systemic sclerosis possible role in vascular inflammation. Egyptian Rheumatol. 33 (2) : 93-98.

12. S. Shenavandeh, T. Tarakemeh, E.K. Sarvestani, et al. (2017). Serum vascular endothelial growth factor (VEGF), soluble VEGF receptor-1 (sVEGFR1) and SVEGFR-2 in systemic sclerosis patients: relation to clinical manifestations and capillaroscopy findings. Egypt Rheumatol. 39 (1): 19-24

13. Mortiz F, Schniering J, Distler JWH, et al. (2017). Tie2 as novel key factor of microangiopathy in systemic sclerosis. Arthritis research \& therapy. 19(1):105.

14. Rosen Y, Diach J, Soliman I, et al. (2016). Vitamin D and autoimmunity. Scand J Rheumatol. 45: 439-447.

15. Bivona G, Agnello L, Pivetti A, et al. (2016). Association between hypovitaminosis $D$ and systemic sclerosis: true or fake? Clin Chim Acta. 458: 115-119.

16. Hax V, Gasparin AA, Schneider L, et al (2019). Vitamin D and Cytokine Profilesin Patients with Systemic Sclerosis. Journal Clin Rheumatol.00:00

17. Atteritano M, Sorbara S, Bagnato G et al (2013). Bone mineral density, bone turnover markers and fractures in patients with systemic sclerosis: a case control study. PLoS One. 8:6-11.

18. Van den Hoogen F, Khanna D, Fransen J, et al (2013). 2013 Classification criteria for systemic sclerosis: an American College of Rheumatology/ European League against Rheumatism collaborative initiatine. Arthritis Rheum. 65:27372747.

19. LeRoy EC, Black C, Fleischmajer R et al (1988). Scleroderma (systemic sclerosis): classification,subsets and pathogenesis. J Rheumatol. 15:202-205.

20. Clements P, Lachenbruch P, Siebold J et al (1995). Inter and intraobserver variability of total skin thickness score (modified Rodnan TSS) in systemic sclerosis. J Rheumatol. 22:1281-1285.

21. Maeda SS, Borba VZ, Camargo MB et al (2014). Recommendations of the Brazilian Society OF 
Endocrinology and Metabology (SBEM) for thediagnosis and treatment of hypovitaminosis D. Arq Bras Endocrinol Metabol.58:411-433.

22. Avouac J, Vallucci M, Smith V et al (2013). Correlations between angiogenic factors and capillaroscopic patterns in systemic sclerosis. Arthritis Res Ther.15(2):R55.

23. Maurizio Cutolo, Carmen Pizzorni, Maria Elena Secchi, et al. (2008). Capillaroscopy. 22(6):1093108.

24. Denton CP, Hughes M, Gak N, et al (2016). BSR andBHPR guideline for the treatment of systemic sclerosis. Rheumatology.55(10):1906-10.

25. CorradoA, Colia R, Mele A, et al. (2015). Relationship between body mass composition, bone mineral density, skin fibrosis and $25(\mathrm{OH})$ Vitamin D serum levels in systemic sclerosis. PLoS One. 10:1-13.

26. Vacca A, Cormier C, Pirias M, et al. (2009). Vitamin $D$ deficiency and insufficiency in 2 independent cohorts of patients with systemic sclerosis.J Rheumatol. 36:1924-1929.

27. Avouac J, Wipff J, Goldman O et al (2008). Angiogenesis in Systemic Sclerosis Impaired Expression of Vascular endothelial growthfactor Receptor 1 in Enothelial Progenitor Dervied Cells Under Hypoxic Conditions. Arthritis and rheumatism. 58(11):3550-61.
28. Matsushita T, Hasegawa M, Hamaguchi $Y$, et al. (2006). Longitudinal analysis of serum cytokine concentrations in systemic sclerosis: association of interleukin 12 elevation with spontaneous regression of skin sclerosis. J Rheumatol. 33:275284.

29. Koenig M, Joyal F, Fritzler MJ et al (2008). Autoantibodies and microvasular damage are independent predictive factors for the progression of Raynaud's phenomenon to systemic sclerosis: a twenty-year prosective study of 586 patients with validation of proposed criteria for early systemic sclerosis. Arthritis and rheumatism.58(12):3902-12.

30. Semenza GL (2012). Hypoxia-inducible factors in physiology and medicine. Cell. 148(3):399-408.

31. Maurer B, Distler A, Suliman YA et al (2014). Vascular endothelial growth factor aggravates fibrosis and vasculopathy in experimental models of systemic sclerosis. Ann Rheum Dis. 73(10):1880-7.

32. Dantas AT, Almeida AR, Sampaio MCPD et al (2018). Different profile of cytokine production in patients with systemic sclerosis and association with clinical manifestations. Immunol Lett. 198:12-16. 